\title{
Universal critical exponents of nonequilibrium phase transitions from holography
}

\author{
Hua-Bi Zeng ${ }^{1,2, *}$ and Hai-Qing Zhang ${ }^{3, \dagger}$ \\ ${ }^{1}$ Center for Gravitation and Cosmology, Yangzhou University, Yangzhou 225009, China \\ ${ }^{2}$ College of Physics Science and Technology, Yangzhou University, Yangzhou 225009, China \\ ${ }^{3}$ Department of Space Science \& International Research Institute for Multidisciplinary Science, \\ Beihang University, Beijing 100191, China
}

(Received 8 September 2018; published 27 November 2018)

\begin{abstract}
We study the critical exponents in the universal scaling laws of a holographic nonequilibrium steady state near its critical point of phase transition, which is driven by an ac electric field sitting in the boundary of the bulk. The applied electric field drives the initial superconducting state into a nonequilibrium steady state with vanishing condensate as its amplitude is greater than a critical value. In the vicinity of the nonequilibrium critical point, we numerically calculate the six static and one dynamical critical exponents, and find that they have similar values to those in equilibrium systems within numerical errors.
\end{abstract}

DOI: 10.1103/PhysRevD.98.106024

\section{INTRODUCTION}

One of the most intriguing features in equilibrium continuous phase transition is the universal scaling behavior near the critical point, which groups various critical phenomena into universality classes; i.e., systems that lie in one universality class share the same scaling behavior [1]. From the point of view of modern physics, the concept of universality has its origin in the renormalization group [2], that the universality roots in the long-range correlations in the system no matter what the microscopic detail is. However, little is understood about the general aspects of nonequilibrium systems. A full classification of the universality classes in nonequilibrium phase transitions is still lacking because of the violation of the detailed balance [3]; thus, the fluctuation-dissipation theorem cannot be applied $[1,4]$. However, it is believed that even for a system far away from equilibrium, the concepts of scaling and universality can still be applied to the nonequilibrium phase transition. There are many attempts in condensed matter physics trying to explain the power law correlations presented in nonequilibrium dynamics [5]. These studies reveal a very close connection between equilibrium and nonequilibrium critical phenomena. Thus, investigating the similarities or differences between equilibrium and

\footnotetext{
*hbzeng@yzu.edu.cn

thqzhang@buaa.edu.cn
}

Published by the American Physical Society under the terms of the Creative Commons Attribution 4.0 International license. Further distribution of this work must maintain attribution to the author(s) and the published article's title, journal citation, and DOI. Funded by SCOAP ${ }^{3}$. nonequilibrium systems may help us to understand the essence of nonequilibrium dynamics.

Nonequilibrium steady state (NESS) is different from the equilibrium state in that NESS has the presence of fluxes in the systems, either by the boundary condition or by bulk driving fields. A well-known example of NESS is the Rayleigh-Bénard experiment which has the horizontal layers of viscous fluid sitting between two heat baths with temperatures $T_{1}$ and $T_{2}$. For $T_{1}=T_{2}$, this system relaxes into a quiescent equilibrium state while a small difference of the temperature, e.g., a small $\delta T=\left|T_{1}-T_{2}\right|$ will have a NESS since energy flux is flowing through the system. We are going to focus on the NESS regime in nonequilibrium state. Increasing $\delta T$ will make the system have more sophisticated structures which will not be explored in this paper; interested readers may refer to [6].

At equilibrium states, the second-order phase transitions result from the long-range correlations, regardless of the original short-range interactions. Interestingly, there is usually a collective behavior over large scales in a strongly coupled complex system [7]. Therefore, it is of particular interest to study the long-range behaviors of strongly coupled nonequilibrium dynamics, and to see whether it will have similar behavior to the equilibrium long-range correlations. Except for the numerical Monte Carlo simulation [8] and lattice gauge theories [9], strongly coupled field theory is notorious for its difficulty to be solved analytically since perturbative methods are impossible to be implemented in the strongly coupled regime. Fortunately, in recent years, people from high energy physics have invented a holographic approach to study the strongly coupled field theory from the weakly coupled gravity, which is dubbed AdS/CFT correspondence [10]. 
In the limit of large gauge group rank $N_{c}$ and large 't Hooft coupling $\lambda$, the AdS/CFT correspondence can study the strongly coupled field theory from the weakly coupled gravity [10-13]. Its application in equilibrium/nonequilibrium dynamics has been investigated in various ways. For instance, AdS/CFT correspondence has been adopted in the study of hot QCD and strongly coupled quark-gluon plasma [14]; the nonequilibrium dynamics of superconducting order parameter after quench $[15,16]$; topological defects formation in Kibble-Zurek mechanism [17,18]; time evolution of nonlocal entanglement observables [19,20]; energy flows between two heat baths [21], etc. Interested readers may refer to the review papers $[22,23]$.

In this paper, we are going to investigate the scaling laws near the critical point of holographic nonequilibrium steady states, which are driven by a sinusoidal applied ac electric field. The universal scaling laws near the critical point in nonequilibrium dynamics were already partially studied in $[17,18,24,25]$. At the initial time, the state is in a static superconducting phase which can be obtained from the holographic construction of the charged scalar model $[26,27]$. After the initial time, we add a sinusoidal ac electric field on the boundary of the spacetime to drive the system away from the static phase [28]. The superconducting order parameter will decrease dramatically according to the amplitude $E$ of the applied electric field, while we have fixed the frequency of the electric field. Therefore, we can regard the amplitude as the controlling parameter which drives the system away from the initial static state. Eventually, the final state will saturate into a superconducting nonequilibrium steady state or normal steady state, depending on the strength of the amplitude. It is found that there exists a critical value of the amplitude $E_{c}$, beyond which the system will finally become a normal state. Based on this nonequilibrium phase transition, we investigate the scaling laws near the critical point $E_{c}$. We numerically explore the six static critical exponents, i.e., $(\alpha, \beta, \gamma, \delta, \eta, \nu)$ and one dynamical critical exponent $z$. The details of the computation can be found in Sec. III. We found that the critical exponents are numerically consistent with those in mean-field theory within numerical errors, which indicates that the holographic system on the boundary in the large $N$ limit does not have great discrepancy from the mean-field theory. These results match those in the previous holographic studies.

The paper is arranged as follows: The holographic background is constructed in Sec. II, while the numerical computations of the critical exponents are given in Sec. III. We draw the conclusions and discussion in Sec. IV. In the Appendix we briefly review the critical exponents we considered in mean-field theory.

\section{HOLOGRAPHIC NONEQUILIBRIUM PHASE TRANSITION}

The action we adopt is a $U(1)$ gauge field $A_{\mu}$ coupled with a complex scalar field $\Psi$ (for simplicity, we work in the probe limit by ignoring the backreaction of the scalar fields and gauge fields to the gravity),

$S=\int d^{4} x \sqrt{-g}\left(-\frac{1}{4} F_{\mu \nu} F^{\mu \nu}-\left|\partial_{\mu} \Psi-i A_{\mu} \Psi\right|^{2}-m^{2}|\Psi|^{2}\right)$,

where $F_{\mu \nu}=\partial_{\mu} A_{\nu}-\partial_{\nu} A_{\mu}$ is the gauge field strength while $m^{2}$ is the mass square of the scalar field. The gravity background is the neutral $\mathrm{AdS}_{4}$ planar black hole, with the metric in Eddington-Finkelstein coordinates as

$$
d s^{2}=\frac{1}{z^{2}}\left(-f(z) d t^{2}-2 d t d z+d x^{2}+d y^{2}\right),
$$

with $f(z)=1-z^{3}$ (we have scaled the anti-de Sitter radius as $L=1$ ). Therefore, $z=1$ is the location of horizon while $z=0$ is the boundary where the field theory lives. Dynamics of the system is governed by the following time dependent equations of motion:

$$
\begin{gathered}
\partial_{t} \partial_{z} \Phi-i A_{t} \partial_{z} \Phi-\frac{1}{2}\left[f \partial_{z}^{2} \Phi+f^{\prime} \partial_{z} \Phi+i \partial_{z} A_{t} \Phi\right. \\
\left.-z \Phi-A_{x}^{2} \Phi\right]=0, \\
\partial_{t} \partial_{z} A_{t}+2 A_{t}|\Phi|^{2}-i f\left(\Phi^{*} \partial_{z} \Phi-\Phi \partial_{z} \Phi^{*}\right) \\
+i\left(\Phi^{*} \partial_{t} \Phi-\Phi \partial_{t} \Phi^{*}\right)=0, \\
\partial_{t} \partial_{z} A_{x}-\frac{1}{2}\left[\partial_{z}\left(f \partial_{z} A_{x}\right)-2 A_{x}|\Phi|^{2}\right]=0, \\
\partial_{z}\left(\partial_{z} A_{t}\right)-i\left(\Phi^{*} \partial_{z} \Phi-\Phi \partial_{z} \Phi^{*}\right)=0,
\end{gathered}
$$

with the following ansatz: $\Phi=\Psi(t, z) / z, A_{t}=A_{t}(t, z)$, $A_{x}=A_{x}(t, z)$, and $A_{z}=A_{y}=0$. The above four equations, in fact, satisfy the following constraint equation:

$$
\frac{d}{d t} \text { Eq. }(6)-\frac{d}{d z} \text { Eq. }(4) \equiv-2 i\left(\text { Eq. }(3) \times \Phi^{*}-\text { c.c. }\right)
$$

where c.c. represents complex conjugation. The constraint equation (7) actually originates from $\nabla_{\mu} \nabla_{\nu} F^{\mu \nu} \equiv 0$, implying the conservation of current. The asymptotic expansions of the fields near the boundary are (we have set the mass square $m^{2}=-2$ without loss of generality)

$$
\begin{aligned}
\left.\Psi(t, z)\right|_{z \rightarrow 0} & =\Psi^{(1)}(t) z+\Psi^{(2)}(t) z^{2}, \\
\left.A_{\mu}(t, z)\right|_{z \rightarrow 0} & =a_{\mu}(t)+b_{\mu}(t) z .
\end{aligned}
$$

According to the holographic dictionary, $\Psi^{(1)}$ is regarded as the source term of the boundary scalar operator $O$ while $\Psi^{(2)}$ is the vacuum expectation $\langle O\rangle$. The coefficients $a_{\mu}$ and $b_{\mu}$ are corresponding to the velocity $v_{\mu}$ and current $J_{\mu}$ 

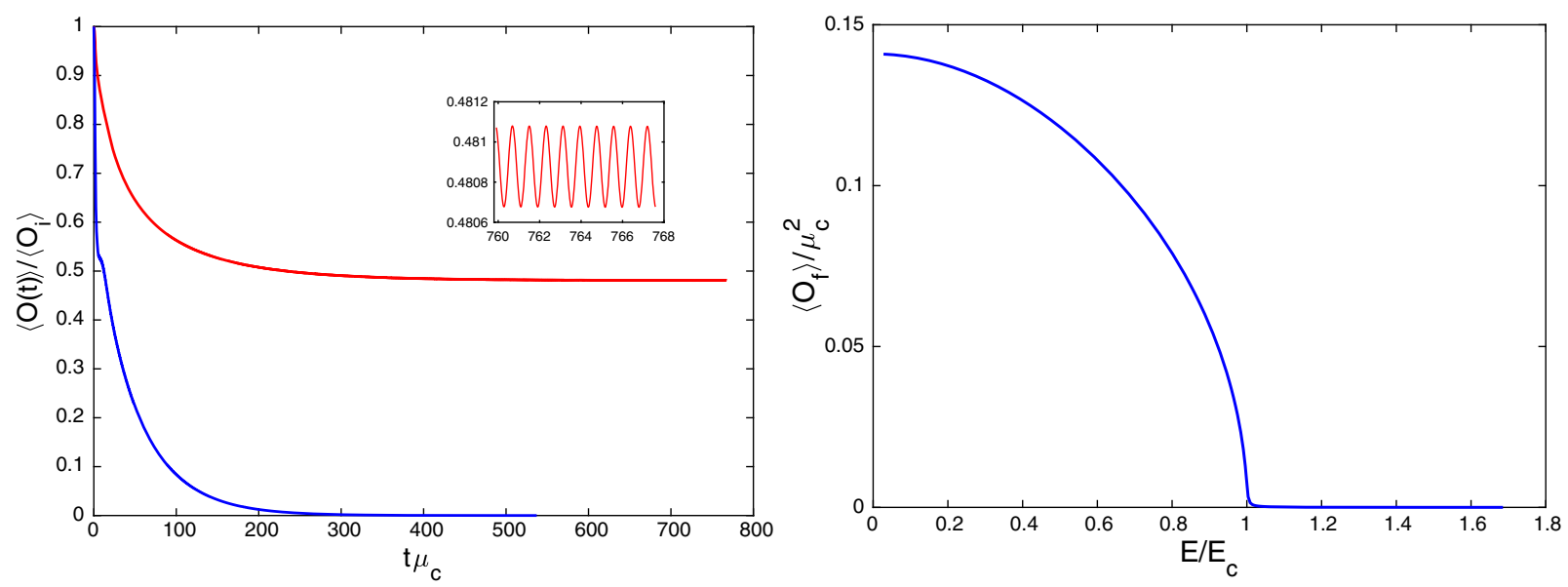

FIG. 1. (Left panel) Time evolution of the ratio between the condensate $\langle O(t)\rangle$ and the initial condensate $\left\langle O_{i}\right\rangle$. The red and blue lines are corresponding to the amplitudes $E=0.8316 E_{c}$ and $E=2.4948 E_{c}$, respectively. The inset plot shows the tiny oscillations of the order parameter for $E=0.8316 E_{c}$ in the late time, which indicates the nonequilibrium steady state. (Right panel) The relation between the final average condensate $\left\langle O_{f}\right\rangle$ in the late time and the amplitude $E$. As $E<E_{c}$ the final state is in the ordered phase with finite condensate $\left\langle O_{f}\right\rangle$, while $E>E_{c}$ the state is in the disordered phase with vanishing condensate. The critical value for the phase transition is $E_{c} \approx 2.1778 \mu_{c}^{2}$.

of the boundary field, respectively. It is worth mentioning that the above four coefficients all depend on time direction as we study the nonequilibrium dynamics of the system.

One may notice that in Eq. (5) the gauge field component $A_{x}$ can vanish independently; however, in order to investigate the nonequilibrium dynamics of the system in response to the external driving force, we turn on $A_{x}$ and impose its $z=0$ boundary condition as [28]

$$
A_{x}(t, z=0)=\frac{E \sin (\Omega t)}{\Omega} .
$$

Thus, on the boundary the electric field along the $x$ direction is $E_{x}(t)=\partial_{t} A_{x}=E \cos (\Omega t)$, in which $E$ and $\Omega$ are the amplitude and frequency of the applied electric field respectively. ${ }^{1}$ In the static case (time-independent), the system has two kinds of phases: One is the disordered phase in high temperature regime without any condensates of the order parameter. The other one is the ordered phase with scalar condensates as the order parameter in the low temperature regime. The temperature of the black hole is $T=3 /\left(4 \pi z_{h}\right)$, in which $z_{h}$ is the horizon and we have scaled it to be $z_{h} \equiv 1$. From static holographic superconductors [27], the critical point for the phase transition is $\mu_{c} \approx 4.07$, thus the critical temperature is $T_{c} \approx 0.06 \mu_{c}$. We assume that the initial condition at $t=0$ is the static solution with a fixed chemical potential $\mu=1.1056 \mu_{c}$; i.e., the system is in the ordered phase/superconducting phase. After the initial time, we then turn on the applied electric field equation (9) to drive the system away from the equilibrium state. The system will finally saturate into a

\footnotetext{
${ }^{1}$ The nonlinear transport coefficients of this model have been intensively studied in [29,30].
}

nonequilibrium steady state after a certain time, depending on the amplitude $E$ and the frequency $\Omega$ of the applied electric field. We need to stress that after a long enough time, the ultimate state is not an equilibrium state, but rather a nonequilibrium steady state which has very tiny steady oscillations in the order parameter because of the sinusoidal applied electric field. From the inset plot of the left panel in Fig. 1, we see that at late times there are tiny steady oscillations of the order parameter compared to its average value, in the order of $\approx 10^{-4}$. Although the final order parameter is not exactly a constant, within the numerical errors we can still use the average value of it to compute the properties of the system [28]. For instance, we can approximately make use of $\langle O(t)\rangle /\left\langle O_{i}\right\rangle \approx 0.4809$, where $\left\langle O_{i}\right\rangle$ is the initial value of the condensate, as the value of the order parameter in the late time for the red line. In the numerics, we fix the frequency of the applied electric field to be $\Omega=3.8594 \mu_{c}$ while varying the amplitude $E$ to drive the system into various nonequilibrium states and ultimately various nonequilibrium steady states. The equations of motion are solved by the fourth order Runge-Kutta method in the $t$ direction and by Chebyshev spectral methods in the $z$ direction.

On the left panel of Fig. 1 we show the time evolution of the condensate for two different amplitudes $E=0.8316 E_{c}$ (the red line) and $E=2.4948 E_{c}$ (the blue line). The vertical axis is the ratio between the condensate $\langle O(t)\rangle$ and the initial static condensate $\left\langle O_{i}\right\rangle$. We see that the condensates decrease quickly as we turn on the applied electric field after $t=0$. The condensate with larger $E$ decreases more rapidly than the one with smaller $E$. In the late time for the nonequilibrium steady state, the condensate will become flat with very tiny oscillations as we have explained above. Therefore, from now on we will use the average value of 
the condensate to represent the late time condensate $\left\langle O_{f}\right\rangle$ of the order parameter. The final condensate $\left\langle O_{f}\right\rangle$ is larger if $E$ is smaller as illustrated in Fig. 1 by the red line and blue line with $\left(E=0.8316 E_{c},\left\langle O_{f}\right\rangle \approx 0.4809\left\langle O_{i}\right\rangle\right)$ and $\left(E=2.4948 E_{c},\left\langle O_{f}\right\rangle \approx 0\right)$, respectively. The right panel of Fig. 1 shows the final condensate $\left\langle O_{f}\right\rangle$ with respect to the amplitude $E$, and we find that the critical point for the phase transition from finite $\left\langle O_{f}\right\rangle$ to vanishing $\left\langle O_{f}\right\rangle$ is around $E_{c} \approx 2.1778 \mu_{c}^{2}$. Therefore, we see that the amplitude parameter $E$ of the applied electric field can literally drive the original superconducting/ordered phase into a normal/ disordered phase, which is a nonequilibrium steady state.

\section{CRITICAL EXPONENTS IN NONEQUILIBRIUM DYNAMICS}

In the equilibrium theory, one can use the critical exponents of the power law to study the critical behavior of the phase transition near the critical point. Usually there are six static critical exponents $(\alpha, \beta, \gamma, \delta, \eta, \nu)$ and one dynamical critical exponents $z$, respectively. ${ }^{2}$ In the Appendix, we briefly review the critical exponents in equilibrium dynamics. It will be interesting to see whether these critical exponents or scaling laws still hold in the nonequilibrium dynamics, or to see how much they will deviate from the equilibrium case. In the following we will study these critical exponents near the critical point of nonequilibrium phase transition from holography, which corresponds to strongly coupled systems on the boundary. As we mentioned above, the amplitude $E$ in Eq. (9) can be regarded as the external source which drives the system away from the critical point $E_{c}$; therefore, the nonequilibrium phase transition will have certain scaling laws with respect to the difference $\epsilon_{E}=1-E / E_{c}$ as we have expected. We numerically calculate these critical exponents in the following subsections. ${ }^{3}$

\section{A. Static critical exponents}

(i) $\alpha=0$ : From the Appendix, the critical exponent $\alpha$ is related to the heat capacity of the system as $C \propto\left|\epsilon_{E}\right|^{-\alpha}=\left|1-E / E_{c}\right|^{-\alpha}$. Following the arguments in [31], we can see that in the disordered phase or the phase with $E>E_{c}$, there is no condensate of the scalar fields. Therefore, the heat capacity of the system is the heat capacity of the black hole. Thus, as we approach the critical point of the nonequilibrium phase transition, the heat

\footnotetext{
${ }^{2}$ One should not confuse the dynamical exponents $z$ with the radial direction $z$ in this paper. Indeed, they can be easily distinguished from the contexts.

${ }^{3}$ The paper [25] also computed the critical exponents of a nonequilibrium phase transition from holography. However, the setup there was different from ours in that they worked in a D3-D7 brane system. Moreover, in their paper the authors did not study the critical exponent $\eta$ and the dynamical critical exponent $z$.
}

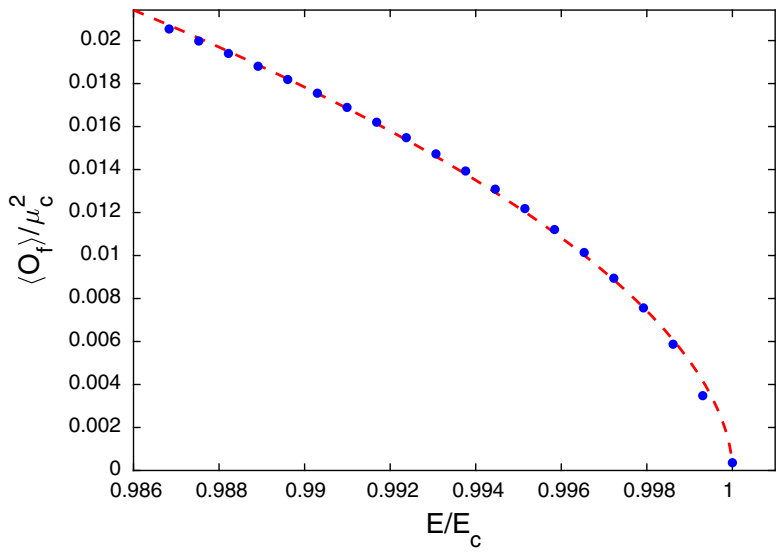

FIG. 2. Relation between $\left\langle O_{f}\right\rangle$ and $E$ in the vicinity of $E_{c}$. The blue dots are the numerical values while the red dashed line is the fitted curve with $\left\langle O_{f}\right\rangle / \mu_{c}^{2} \approx 0.2189 \times\left|1-E / E_{c}\right|^{1 / 2}$.

capacity converges to a constant since we worked in the probe limit. Therefore, we can deduce that $\alpha=0$.

(ii) $\beta=1 / 2$ : The critical exponent $\beta$ can be read off from the relation between the order parameter $\left\langle O_{f}\right\rangle$ with respect to $\epsilon_{E}$ near the critical point such that $\left\langle O_{f}\right\rangle / \mu_{c}^{2} \propto\left|1-E / E_{c}\right|^{\beta}$. By increasing $E$ in Eq. (9), the order parameter in the final nonequilibrium steady state decreases and vanishes at the critical point $E_{c} \approx 2.1778 \mu_{c}^{2}$. Figure 2 shows the relation between $\left\langle O_{f}\right\rangle$ and $E$ near the critical point; the blue dots are the numerical results while the red dashed line is the fitted curve, which is roughly $\left\langle O_{f}\right\rangle / \mu_{c}^{2} \approx 0.2189 \times\left|1-E / E_{c}\right|^{1 / 2}$. The numerical dots and the fitted curve match very well. Therefore, we see that $\beta \sim 1 / 2$, which is the same as the value of $\beta$ in the mean-field theory.

(iii) $\nu=1 / 2$ : To compute the critical exponents $\nu$ we need to solve the perturbative equations of the system, and then we can get the relation between the correlation length $\xi$ and $\epsilon_{E}$, such as $\xi \propto\left|\epsilon_{E}\right|^{-\nu}$. Basically $\nu>0$, which indicates the typical divergence of the correlation length near the critical point. The correlation length $\xi$ can be read off from the correlation function (response function) of the order parameter. In the Fourier space the correlation function reads

$\chi(\omega, k)=\left\langle O(\omega, k) O^{\dagger}(-\omega,-k)\right\rangle \sim \frac{1}{i c \omega+k^{2}+1 / \xi^{2}}$,

where $c$ is a parameter, andn $\omega$ and $k$ are, respectively, the frequency and momentum of the transformed Fourier modes. The poles of the correlation function correspond to the system's quasinormal modes (QNMs) [32], which can depict the relaxation behavior of the system in the late time. As we already mentioned, the system will go to steady state in the late time. Therefore, it is more convenient to approximately treat the background fields as time independent in the late time. In order to study the QNMs of 

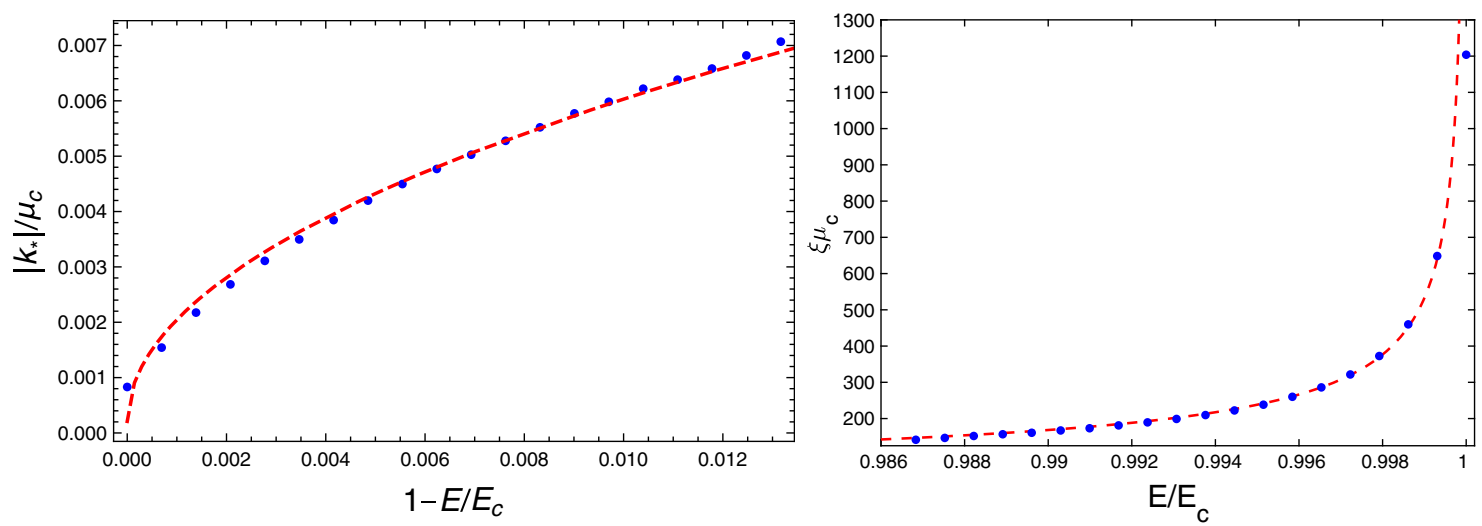

FIG. 3. (Left panel) Relation between the lowest modes of momentum $\left|k_{*}\right|$ and $\left(1-E / E_{c}\right)$ with $\left|k_{*}\right| / \mu_{c} \approx 0.05827 \times\left|1-E / E_{c}\right|^{1 / 2}$. (Right panel) Relation between the correlation length $\xi$ and $E$ with $\xi \mu_{c} \approx 17.1615 \times\left|1-E / E_{c}\right|^{-1 / 2}$. For both plots, the dots are the numerical results while the dashed lines are the fitted curves.

the system, we need to perturb the fields linearly. The first order perturbations of the fields can be written as $\delta A_{t}(t, z, x) \rightarrow e^{-i \omega t+i k x} \delta A_{t}(z)$ and $\delta \Phi(t, z, x) \rightarrow$ $e^{-i \omega t+i k x} \delta \Phi(z){ }^{4}$ The equations of motion for the first order fluctuations of the fields read

$$
\begin{gathered}
\omega \delta A_{t}^{\prime}+i\left(k^{2}+2 \Phi^{2}\right) \delta A_{t}+4 i A_{t} \Phi \delta \Phi=0, \\
\left(i A_{t}^{\prime}-z-\left(A_{x}-k\right)^{2}\right) \delta \Phi+i \Phi \delta A_{t}^{\prime}+2 i \Phi^{\prime} \delta A_{t} \\
+\left(2 i\left(A_{t}+\omega\right)-3 z^{2}\right) \delta \Phi^{\prime}+\left(1-z^{3}\right) \delta \Phi^{\prime \prime}=0
\end{gathered}
$$

where 1 is the derivative with respect to the radial $z$ coordinate. We will calculate the QNMs of the system near the critical point, i.e., $E \sim E_{c}$. Besides, we make use of the average values of the background fields as we calculate the QNMs since the system is in the steady state in the late time as we already mentioned above.

From the poles of the response function in Eq. (10), we see that the relation between the correlation length $\xi$ and the momentum $k$ can be obtained by solving Eqs. (11) and (12) and setting $\omega=0$. Therefore, the poles of the response function are located in $k_{*}^{2}=-1 / \xi^{2}$. By varying the external parameter $E$, we then select the lowest modes of $k$ which have the negative imaginary parts mostly closing to the real axis, for instance, the mode with $E=0.9868 E_{c}$, the lowest $k=-0.007068 i \mu_{c}$. From the left panel of Fig. 3 we find that the fitted curve for the numerical data is roughly $\left|k_{*}\right| / \mu_{c} \approx 0.05827 \times\left|1-E / E_{c}\right|^{1 / 2}$ within numerical errors. Therefore, the correlation length $\xi=1 /\left|k_{*}\right| \propto\left|\epsilon_{E}\right|^{-1 / 2}$, which is shown on the right panel of Fig. 3. Thus, we see that near the critical point of the nonequilibrium phase

\footnotetext{
${ }^{4}$ We did not perturb the field $A_{x}$ when we calculated the QNMs, since physically $A_{x}$ plays the role of the external driving force in the system. On the other hand, QNMs are the first order perturbations which respond to the system. Therefore, it is more physical to treat $A_{x}$ as the background field which does not contribute fluctuating modes to QNMs.
}

transition $\nu=1 / 2$, which is the same as the one in the equilibrium field theory.

(iv) $\delta=3$ : The critical exponent $\delta$ can be obtained from the relation between the order parameter and its source term near the critical point, i.e., $\left.\langle O\rangle\right|_{E \sim E_{c}}=\Psi^{(2)} \propto\left(\Psi^{(1)}\right)^{1 / \delta}[31]$. As we calculate $\delta$, we set $E$ very close to $E_{c}$ and slightly vary the source $\Psi^{(1)}$ away from zero since we still roughly need the condensate of the order parameter from the spontaneous symmetry breaking. So we keep the source term $\Psi^{(1)}$ in the order of $10^{-4} \mu_{c}$ which can be seen in Fig. 4. In Fig. 4 we plot the relation between the condensate value of the order parameter and the source; the dots are the numerical results while the dashed line is the fitted curve. Within numerical errors, we find that $\langle O\rangle /\left.\mu_{c}^{2}\right|_{E_{c}} \approx 0.2674 \times\left(\Psi^{(1)} / \mu_{c}\right)^{1 / 3}$, thus $\delta=3$ as one expected in the equilibrium dynamics.

(v) $\gamma=1$ : The critical exponent $\gamma$ can be read off from $\chi(\omega=0, k=0) \propto\left|1-E / E_{c}\right|^{-\gamma}$. As we know, in the AdS/CFT correspondence the response function is

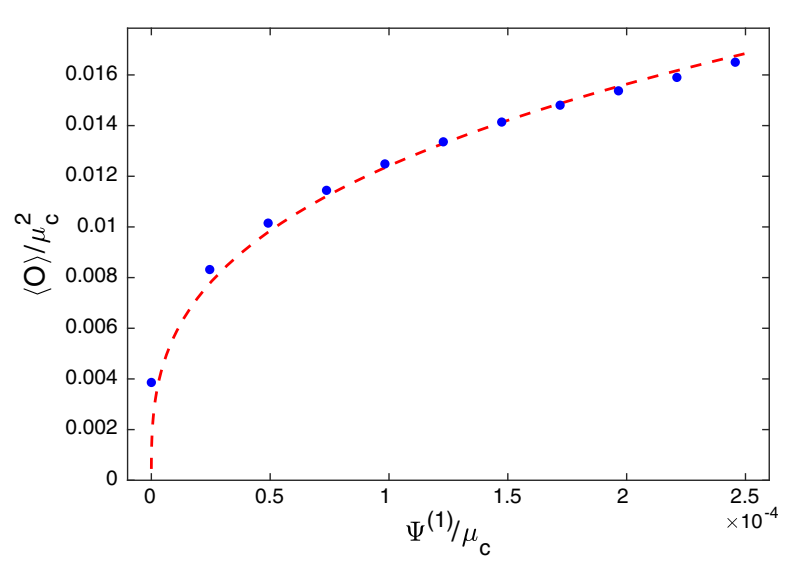

FIG. 4. Relation between the order parameter and the source, from which we can deduce $\delta=3$. Dots are the numerical results while the dashed line is the fitted curve, which has relation $\langle O\rangle / \mu_{c}^{2} \approx 0.2674 \times\left(\Psi^{(1)} / \mu_{c}\right)^{1 / 3}$. 


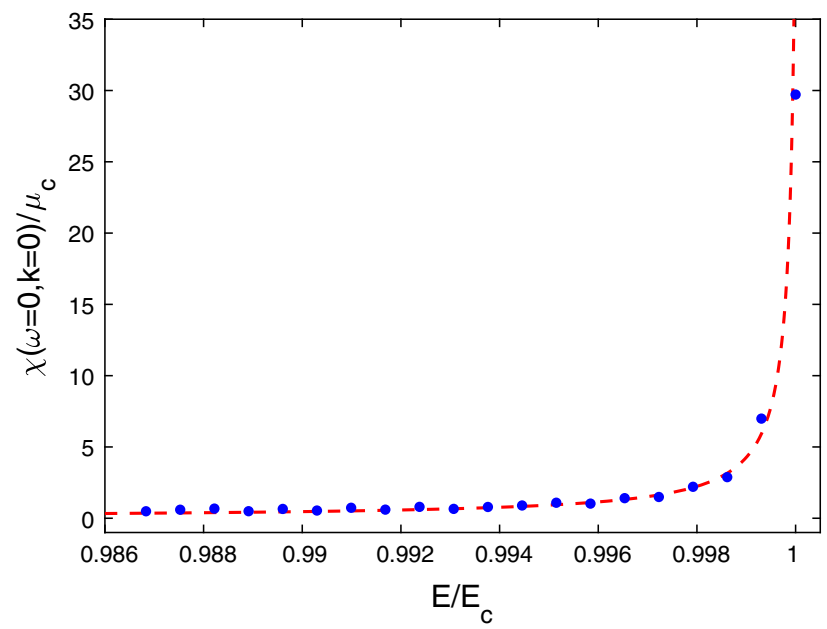

FIG. 5. Relation between the response function $\chi(\omega=0$, $k=0)$ and $E$, from which we can deduce $\gamma=1$. The dots are the numerical results while the dashed line is the fitted curve with the relation $\chi(\omega=0, k=0) / \mu_{c} \approx 0.004692 \times\left|1-E / E_{c}\right|^{-1}$.

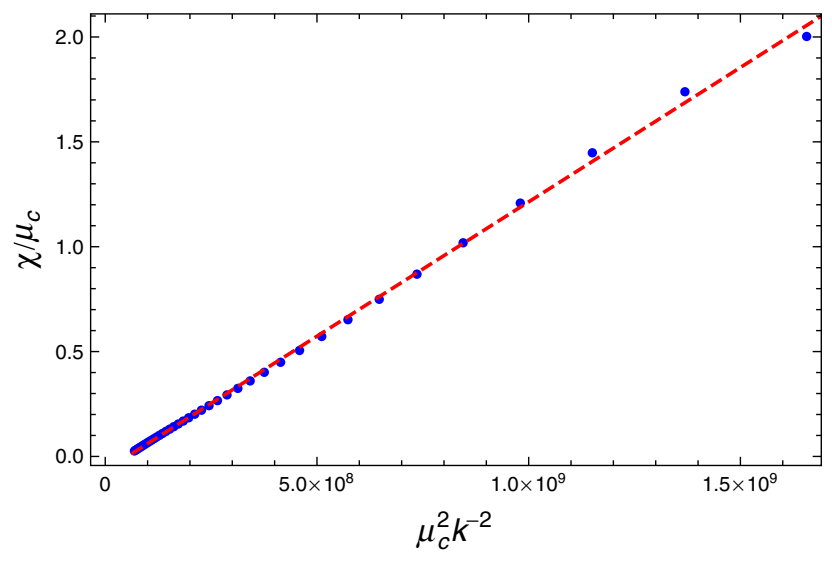

FIG. 6. Relation between the response function $\chi$ and $k^{-2}$, from which one can deduce that $\eta=0$. The dots are the numerical results while the dashed line is the fitted curve with the relation $\chi / \mu_{c} \approx 1.2801 \times 10^{-9} \mu_{c}^{2} k^{-2}$.
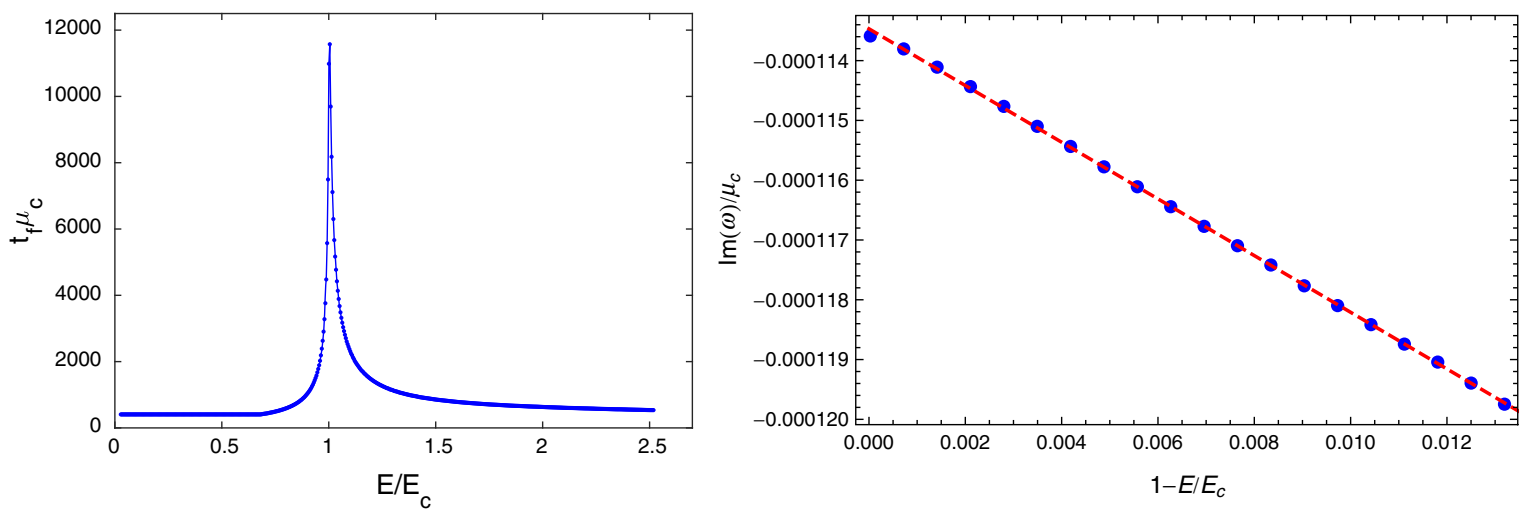

FIG. 7. (Left panel) Relation between the saturation time $t_{f}$ and the amplitude $E$. $t_{f}$ diverges near the critical point $E_{c}$, which indicates the critical slowing down near the critical point. (Right panel) Linear relation between the imaginary part of the QNMs and $\left|1-E / E_{c}\right|$, from which we can deduce the relation between relaxation time with respect to $\left|1-E / E_{c}\right|$ and hence $z=2$. The dots are the numerical results while the dashed line is the fitted curve with $\operatorname{Im}(\omega) / \mu_{c} \approx-4.7409 \times 10^{-4}\left|1-E / E_{c}\right|$.

obtained from $\chi(\omega=0, k=0)=\delta \psi_{2} / \delta \psi_{1}$, where $\delta \psi_{2}$ and $\delta \psi_{1}$ are the perturbations of the order parameter and the source, respectively. In the numerical computation we vary the amplitude of the electric field $E$ while fixing $\left.\delta \psi_{1}\right|_{z=1}=0.2457 \mu_{c}$, and then study the ratio $\delta \psi_{2} / \delta \psi_{1}$ on the $z=0$ boundary. From Fig. 5 we can fit $\chi(\omega=0$, $k=0) / \mu_{c} \approx 0.004692 \times\left|1-E / E_{c}\right|^{-1}$; thus, within numerical errors $\gamma=1$ is the same as that in the equilibrium field theory.

(vi) $\eta=0$ : From $\chi(\omega=0, k) \propto k^{\eta-2}$ one can read off the value of the critical exponent $\eta$. Therefore, in the vicinity of the critical point $E_{c}$, we slightly change the momentum $k$ (in the order of $10^{-4} \mu_{c}$ ) away from zero to calculate the response function $\chi$. The results are shown in the Fig. 6 . The dots are the numerical results while the dashed line is the fitted curve. Therefore, from Fig. 6 we see that $\chi$ is linear proportional to $k^{-2}$ with $\chi / \mu_{c} \approx 1.2801 \times 10^{-9} \mu_{c}^{2} k^{-2}$, which implies $\eta=0$. This result of $\eta$ is similar to that in equilibrium dynamics.

Therefore, from the above numerical results of the six static critical exponents we find that they have similar values compared to those in equilibrium dynamics, i.e., $(\alpha, \beta, \gamma, \delta, \nu, \eta)=\left(0, \frac{1}{2}, 1,3, \frac{1}{2}, 0\right)$. Moreover, they also satisfy the four identities (A2), (A3), (A4), and (A5) as shown in the Appendix.

\section{B. Dynamical critical exponent $z=2$}

After certain time $t_{f}$, the system will saturate into the nonequilibrium steady states as we discussed above. ${ }^{5}$ In order to find the approximate value of the saturation

\footnotetext{
${ }^{5}$ Let us call $t_{f}$ saturation time loosely. It will approximately equal the relaxation time which will be defined from the QNMs exactly in the following.
} 
time $t_{f}$, we set a threshold that if $\langle O(t+\Delta t)\rangle /\langle O(t)\rangle \lesssim 10^{-5}$, where $\Delta t$ is the periodicity of the order parameter in the steady state, we can say that the time $t$ is approximately the saturation time $t_{f}$ (please refer to the left panel of Fig. 1 that $\Delta t \mu_{c} \approx 0.8$ for the red curve). We show the relation between $t_{f}$ and the external amplitude $E$ in the left panel of Fig. 7. Near the critical point $E_{c}$, we can see the divergence of the saturation time $t_{f}$, which indicates the critical slowing down near the phase transition point.

Dynamical critical exponent $z$ can be read off from the relation $\tau(k=0) \propto \xi^{z}$, where $\tau$ is the relaxation time. Since we already knew $\xi \propto\left|1-E / E_{c}\right|^{-1 / 2}$, we need to check the relation between $\tau$ and $\left|1-E / E_{c}\right|$ by varying $E$, hence $\tau(k=0) \propto\left|1-E / E_{c}\right|^{-z / 2}$. From the fact that the relaxation time is related to the inverse of the imaginary part of the QNMs $\omega$ in Eq. (10), we can compute the QNMs with respect to $\left|1-E / E_{c}\right|$ while fixing $k=0$. In the right panel of Fig. 7 we show the linear relation between the imaginary parts of the lowest modes of the QNMs and $\left(1-E / E_{c}\right)$. The relation is roughly $\operatorname{Im}(\omega) / \mu_{c} \approx-4.7409 \times 10^{-4}\left|1-E / E_{c}\right|$, therefore, $\tau=1 / \operatorname{Im}(\omega) \propto\left|1-E / E_{c}\right|^{-1}$ (the lowest modes of $\omega$ have negative imaginary parts indicate that the system is stable against perturbations). Hence, we get $\tau \propto \xi^{2}$ and $z=2$ which is the same as that in the equilibrium field theory. This also indicates that our nonequilibrium system belongs to the A model defined in [1] and satisfies $z=2-\eta$ as well.

\section{CONCLUSIONS AND DISCUSSIONS}

We systematically studied the critical exponents of the universal scaling laws near the critical point of a holographic nonequilibrium phase transition, which was driven by an ac electric field sitting in the boundary of the bulk. The final states entered into a nonequilibrium steady state, rather than an equilibrium state, due to the external periodic electric field. In the final steady state, we ignored the tiny oscillations of the order parameters and took advantage of their average values in our numerics. By varying the amplitude $E$ of the applied ac electric field, we found that beyond a critical value of the amplitude $E_{c}$, the initial superconducting phase would be dramatically destroyed into a normal state with average vanishing condensate. We numerically calculated the six static and one dynamical critical exponents near this nonequilibrium phase transition critical point. It was found that these critical exponents had similar values compared to their counterparts in equilibrium dynamics, i.e., $(\alpha, \beta, \gamma, \delta, \nu, \eta)=$ $\left(0, \frac{1}{2}, 1,3, \frac{1}{2}, 0\right)$ and $z=2$. Therefore, these exponents also satisfy the four identities (A2), (A3), (A4), and (A5) as shown in the Appendix. This result was consistent with previous studies in holography that the holographic superconductors behave as a mean-field theory. The reason may be that the scaling laws near the critical point are a largescale behavior, which ignores the short-range or quantum properties of the system. Besides, the large $N_{c}$ limit of the
AdS/CFT correspondence will suppress the quantum effect of the boundary field theory although it is strongly coupled. Therefore, in the long-range limit the universal scaling laws look alike between the equilibrium and nonequilibrium dynamics.

In this paper we only considered the effects of the amplitude $E$ to the phase transition as well as the critical exponents. It will be interesting to see whether the frequency $\Omega$ will have similar effects to the critical exponents. Indeed, from our tentative computation we find that the condensate of the order parameter will behave similarly to that in meanfield theory, such as $\left\langle O_{f}\right\rangle \propto\left|\epsilon_{\kappa}\right|^{1 / 2}$, where $\epsilon_{\kappa}=1-\kappa / \kappa_{c}$ and $\kappa \equiv \mu_{c} / \Omega$. Therefore, one can deduce that the static critical exponent $\beta=1 / 2$ if we regard frequency as a controlling parameter. The interesting thing is that from the above relation $\left\langle O_{f}\right\rangle \propto\left|\epsilon_{\kappa}\right|^{1 / 2}$ one finds that if $\Omega>\Omega_{c}$ (where $\Omega_{c}=\mu_{c} / \kappa_{c}$ ) the system will remain in the superconducting phase while on the contrary $\Omega<\Omega_{c}$ the system will be in the normal state with vanishing condensate. This counterintuitive phenomenon actually can be explained by the "Wyatte-Dayem" effect in condensed matter physics [33], where higher frequency fields will enhance the superconductivity $[34,35]$. From the tentative result $\beta=1 / 2$ we expect that other critical exponents, such as $(\alpha, \gamma, \delta$, $\nu, \eta)$ and $z$, will have similar values to those in mean-field theory. We will leave this interesting topic as a future work.

\section{ACKNOWLEDGMENTS}

H. B.Z. and H.Q.Z. are supported by the National Natural Science Foundation of China (Grants No. 11675140, No. 11705005, and No. 11875095).

\section{APPENDIX: POWER-LAW SCALING AND CRITICAL EXPONENTS IN EQUILIBRIUM DYNAMICS}

In the equilibrium dynamics, there are six static critical exponents $(\alpha, \beta, \gamma, \delta, \nu, \eta)$ and one dynamical critical exponent $z$. The static critical exponents, such as in the ferromagnetic phase transition, can be obtained from the following definitions [36]:

$$
\begin{aligned}
& C \propto\left|\epsilon_{T}\right|^{-\alpha}, \quad M \propto\left|\epsilon_{T}\right|^{\beta}, \quad \chi \propto\left|\epsilon_{T}\right|^{-\gamma}, \\
& M \propto|h|^{1 / \delta}, \quad \chi \propto e^{-r / \xi} \propto r^{2-d-\eta} \propto k^{\eta-2}, \\
& \xi \propto\left|\epsilon_{T}\right|^{-\nu},
\end{aligned}
$$

in which $\epsilon_{T}=\left(T_{c}-T\right) / T_{c}$ is the reduced temperature, $C$ is the heat capacity, $M$ is the magnetization, $\chi$ is the static susceptibility, $h$ is the external magnetic field, $d$ is the spatial dimension, $k$ is the momentum of the modes, and $\xi$ is the correlation length of the order parameter. In the meanfield theory, the six critical exponents satisfy the following relations:

$$
\alpha+2 \beta+\gamma=2 \quad \text { (Rushbrooke) }
$$




$$
\begin{aligned}
\gamma & =\beta(\delta-1) \quad(\text { Widom }), \\
\gamma & =\nu(2-\eta) \quad(\text { Fisher }), \\
2-\alpha & =d \nu \quad \text { (Josephson). }
\end{aligned}
$$

Normally, the critical exponents in the equilibrium dynamics are $(\alpha, \beta, \gamma, \delta, \nu, \eta)=\left(0, \frac{1}{2}, 1,3, \frac{1}{2}, 0\right)$.

In the dynamical case, we will only focus on the model A equilibrium system. From [1], the dynamical exponents $z$ can be obtained from $\tau \propto \xi^{z}$ where $\tau$ is the relaxation time. For the model $\mathrm{A}, z=2-\eta$.
[1] P. C. Hohenberg and B. I. Halperin, Theory of dynamic critical phenomena, Rev. Mod. Phys. 49, 435 (1977).

[2] K. G. Wilson, Renormalization group and critical phenomena. I. Renormalization group and the Kadanoff scaling picture, Phys. Rev. B 4, 3174 (1971); Renormalization group and critical phenomena. II. Phase-space cell analysis of critical behavior, Phys. Rev. B 4, 3184 (1971).

[3] H. Hinrichsen, Non-equilibrium phase transitions, Physica A (Amsterdam) 369, 1 (2006).

[4] Z. Rácz, Nonequilibrium phase transitions, in Lecture Notes for Troisieme Cycle Lectures (2004), http://cgl.elte.hu/ $\sim$ racz/Trois-04-notes.pdf.

[5] G. Odor, Universality classes in nonequilibrium lattice systems, Rev. Mod. Phys. 76, 663 (2004).

[6] M. C. Cross and P. C. Hohenberg, Pattern formation outside of equilibrium, Rev. Mod. Phys. 65, 851 (1993).

[7] M. Henkel, H. Hinrichsen, and S. Lübeck, Non-Equilibrium Phase Transitions (Springer Science \& Business Media, The Netherlands, 2008), Vol. I, II.

[8] K. Binder, The Monte Carlo Method in Condensed Matter Physics (Springer, New York, 1995).

[9] H. J. Rothe, Lattice Gauge Theories, An Introduction, 4th ed. (World Scientific, Singapore, 2012).

[10] J. M. Maldacena, The large N limit of superconformal field theories and supergravity, Int. J. Theor. Phys. 38, 1113 (1999); Adv. Theor. Math. Phys. 2, 231 (1998).

[11] S. S. Gubser, I. R. Klebanov, and A. M. Polyakov, Gauge theory correlators from noncritical string theory, Phys. Lett. B 428, 105 (1998).

[12] E. Witten, Anti-de Sitter space and holography, Adv. Theor. Math. Phys. 2, 253 (1998).

[13] O. Aharony, S. S. Gubser, J. M. Maldacena, H. Ooguri, and Y. Oz, Large $\mathrm{N}$ field theories, string theory and gravity, Phys. Rep. 323, 183 (2000).

[14] J. Casalderrey-Solana, H. Liu, D. Mateos, K. Rajagopal, and U. A. Wiedemann, Gauge/String Duality, Hot QCD and Heavy Ion Collisions (Cambridge University Press, Cambridge, UK, 2014).

[15] K. Murata, S. Kinoshita, and N. Tanahashi, Non-equilibrium condensation process in a holographic superconductor, J. High Energy Phys. 07 (2010) 050.

[16] M. J. Bhaseen, J. P. Gauntlett, B. D. Simons, J. Sonner, and T. Wiseman, Holographic Superfluids and the Dynamics of Symmetry Breaking, Phys. Rev. Lett. 110, 015301 (2013).

[17] P. M. Chesler, A. M. Garcia-Garcia, and H. Liu, Defect Formation beyond Kibble-Zurek Mechanism and Holography, Phys. Rev. X 5, 021015 (2015).
[18] J. Sonner, A. del Campo, and W. H. Zurek, Universal far-from-equilibrium dynamics of a holographic superconductor, Nat. Commun. 6, 7406 (2015).

[19] X. Bai, B. H. Lee, L. Li, J. R. Sun, and H. Q. Zhang, Time evolution of entanglement entropy in quenched holographic superconductors, J. High Energy Phys. 04 (2015) 066.

[20] J. Erdmenger, D. Fernandez, M. Flory, E. Megias, A. K. Straub, and P. Witkowski, Time evolution of entanglement for holographic steady state formation, J. High Energy Phys. 10 (2017) 034.

[21] M. J. Bhaseen, B. Doyon, A. Lucas, and K. Schalm, Energy flow in quantum critical systems far from equilibrium, Nat. Phys. 11, 509 (2015).

[22] V. E. Hubeny and M. Rangamani, A holographic view on physics out of equilibrium, Adv. High Energy Phys. 2010, 1 (2010).

[23] P. M. Chesler and L. G. Yaffe, Numerical solution of gravitational dynamics in asymptotically anti-de Sitter spacetimes, J. High Energy Phys. 07 (2014) 086.

[24] S. Nakamura, Nonequilibrium Phase Transitions and Nonequilibrium Critical Point from AdS/CFT, Phys. Rev. Lett. 109, 120602 (2012).

[25] M. Matsumoto and S. Nakamura, Critical exponents of nonequilibrium phase transitions in AdS/CFT correspondence, arXiv:1804.10124 [Phys. Rev. D (to be published)].

[26] S. S. Gubser, Breaking an Abelian gauge symmetry near a black hole horizon, Phys. Rev. D 78, 065034 (2008).

[27] S. A. Hartnoll, C. P. Herzog, and G. T. Horowitz, Building a Holographic Superconductor, Phys. Rev. Lett. 101, 031601 (2008).

[28] W. J. Li, Y. Tian, and H. b. Zhang, Periodically driven holographic superconductor, J. High Energy Phys. 07 (2013) 030.

[29] H. B. Zeng, Y. Tian, Z. Y. Fan, and C. M. Chen, Nonlinear transport in a two dimensional holographic superconductor, Phys. Rev. D 93, 121901 (2016).

[30] H. B. Zeng, Y. Tian, Z. Fan, and C. M. Chen, Nonlinear conductivity of a holographic superconductor under constant electric field, Phys. Rev. D 95, 046014 (2017).

[31] K. Maeda, M. Natsuume, and T. Okamura, Universality class of holographic superconductors, Phys. Rev. D 79, 126004 (2009).

[32] P. K. Kovtun and A. O. Starinets, Quasinormal modes and holography, Phys. Rev. D 72, 086009 (2005).

[33] A. F. G. Wyatt, V. M. Dmitriev, W. S. Moore, and F. W. Sheard, Microwave-Enhanced Critical Supercurrents in 
Constricted Tin Films, Phys. Rev. Lett. 16, 1166 (1966); A. H. Dayem and J. J. Wiegand, Behavior of thin-film superconducting bridges in a microwave field, Phys. Rev. 155, 419 (1967).

[34] G. M. Eliashberg, Film superconductivity stimulated by a high-frequency field, JETP Lett. 11, 114 (1970).
[35] M. Tinkham, Introduction to Superconductivity, 2nd ed. (McGraw-Hill Inc. Press, U.S., 1996).

[36] C. Itzykson and J.-M. Drouffe, Statistical Field Theory. Vol. 1: From Brownian Motion to Renormalization and Lattice Gauge Theory (Cambridge University Press, Cambridge, England, 1989). 